See discussions, stats, and author profiles for this publication at: https://www.researchgate.net/publication/303890544

\title{
Quantifying Nonlinear Contributions to Cortical Responses Evoked by Continuous Wrist Manipulation
}

Article in IEEE Transactions on Neural Systems and Rehabilitation Engineering • May 2017

DOI: 10.1109/TNSRE.2016.2579118

CITATIONS

5

5 authors, including:

Martijn Vlaar

Delft University of Technology

8 PUBLICATIONS 21 CITATIONS

SEE PROFILE

$+3$

Alistair Neil Vardy

Amsterdam University of Applied Sciences/Centre for Applied Research on Education

17 PUBLICATIONS 66 CITATIONS

SEE PROFILE

Some of the authors of this publication are also working on these related projects:

Project Human gait, stumble and... fall? View project

Project $\quad$ Citius Altius Sanius - Injury free exercise for everyone View project
READS

49

Teodoro Solis-Escalante

Radboud University Medical Centre (Radboudumc)

42 PUBLICATIONS 1,890 CITATIONS

SEE PROFILE

Frans CT van der Helm

Delft University of Technology

297 PUBLICATIONS 10,882 CITATIONS

SEE PROFILE 


\title{
Quantifying Nonlinear Contributions to Cortical Responses Evoked by Continuous Wrist Manipulation
}

\author{
Martijn P. Vlaar, Student Member, IEEE, Teodoro Solis-Escalante, Member, IEEE, Alistair N. Vardy, \\ Frans C. T. van der Helm, and Alfred C. Schouten
}

\begin{abstract}
Cortical responses to continuous stimuli as recorded using either magneto- or electroencephalography (EEG) have shown power at harmonics of the stimulated frequency, indicating nonlinear behavior. Even though the selection of analysis techniques depends on the linearity of the system under study, the importance of nonlinear contributions to cortical responses has not been formally addressed. The goal of this paper is to quantify the nonlinear contributions to the cortical response obtained from continuous sensory stimulation. EEG was used to record the cortical response evoked by continuous movement of the wrist joint of healthy subjects applied with a robotic manipulator. Multisine stimulus signals (i.e. the sum of several sinusoids) elicit a periodic cortical response and allow to assess the nonlinear contributions to the response. Wrist dynamics (relation between joint angle and torque) were successfully linearized, explaining $99 \%$ of the response. In contrast, the cortical response revealed a highly nonlinear relation; where most power $(\sim 80 \%)$ occurred at nonstimulated frequencies. Moreover, only $10 \%$ of the response could be explained using a nonparametric linear model. These results indicate that the recorded evoked cortical responses are governed by nonlinearities and that linear methods do not suffice when describing the relation between mechanical stimulus and cortical response.
\end{abstract}

Index Terms - EEG, EMG, nonlinear, neuromuscular control, system identification

\section{INTRODUCTION}

$\mathrm{S}$ ENSORY feedback is crucial for effective motion control and allows compensating for internal and external disturbances. For example, proprioceptors in the human body,

Paper submitted for review on June 10, 2015. This research was funded by the European Research Council under the European Union's Seventh Framework Programme (FP/2007-2013) ERC Grant Agreement n. 291339, project 4DEEG: A new tool to investigate the spatial and temporal activity patterns in the brain.

M. P. Vlaar, T. Solis-Escalante, A. N. Vardy, F. C. T. van der Helm, and A. C. Schouten are with the BioMechanical Engineering Department, Faculty of Mechanical, Maritime and Materials Engineering, Delft University of Technology, Delft, The Netherlands (e-mail: m.p.vlaar@tudelft.nl).

F. C. T. van der Helm and A. C. Schouten are also with the Department of Physical Therapy and Human Movement Sciences, Feinberg School of Medicine, Northwestern University, Chicago, USA

A. C. Schouten is also with the Laboratory of BioMechanical Engineering, MIRA Institute for Biomedical Technology and Technical Medicine, University of Twente, Enschede, The Netherlands such as muscle spindles and Golgi tendon organs, provide sensory feedback on the state of limbs (i.e., position, velocity, and force). Reflexive control action can originate from spinal level (short latency) and from supra-spinal level (long latency) [1]. Disturbed sensory function or sensorimotor integration is often implicated in movement disorders [2]. The functioning of the somatosensory system can be assessed by applying sensory stimuli and studying muscle or brain response [3-5].

Sensory stimuli are commonly presented as transients; such as when investigating the patellar stretch reflex. The response of a muscle to such a stretch can be recorded using electromyography (EMG)[6]. The dynamic cortical response to a transient sensory stimulus can be noninvasively recorded using magnetoencephalography or electroencephalography (EEG) and is referred to as the event related field or event related potential (ERP). Investigating the sensorimotor system with intermittent short lasting stimuli only reveals the transient response of the system. As an alternative, continuous stimuli, such as sinusoidal, square wave or noise-like signals, are capable of continuously engaging the system in the processing of information; therefore revealing both transient and steadystate behavior [7]. While transient responses depend on initial conditions, steady-state responses present the system behavior accommodated to the stimulation and regardless of the initial state.

Several studies used continuous mechanical stimuli to investigate intrinsic and reflexive limb dynamics by recording the mechanical and muscle response during postural control tasks [8-10]. The role of the cortical structures in reflexive feedback control is yet still not fully understood [1]. Cortical sensory processing of continuous mechanical stimulation has been investigated, for example using vibrotactile stimulation to the fingers, hand and foot [11-13].

Increased insight in normal and pathological sensorimotor function can be obtained by modelling the relation between stimulus and response. To work towards developing these models it is essential to determine which model classes are appropriate. Studies using continuous vibrotactile stimulation report responses at frequencies other than the frequencies present in the stimulus, illustrating a nonlinear relationship between the stimulus and the cortical response measured by EEG [14-17]. Mechanical (i.e. joint angle and torque) and EMG recordings obtained from continuous mechanical 
stimulation also show a small response at non-stimulated frequencies [18-20]. Nonlinear responses in the sensorimotor system could result from sensors (e.g. unidirectional sensitivity), muscles (e.g. unidirectional force generation and a nonlinear force-length relationship) and other parts of the sensorimotor system including the central nervous system.

Although many studies acknowledge the nonlinear properties of the cortical response to sensory stimuli, it remains unclear to which degree the nonlinearities govern the response. Systems with weak nonlinear behavior can be studied in a specific operating range, facilitating linear analysis. Linear system identification techniques are matured, computationally undemanding, require little a priori knowledge and can reveal many characteristics of the system under study, including time delays. Linear techniques are useful if the system under study can be properly linearized, which should be checked during analysis. If the linear approximation only describes a small portion of the behavior of the system, any conclusions based on the linearized system will most likely not be applicable to the actual system under study. The well-established and accessible linear system identification framework should in this case be exchanged for a nonlinear system identification approach. Applying system identification to systems with strong nonlinear behavior requires techniques which are computationally more demanding and often require a priori selection of a model structure or order.

To determine which analysis tools are appropriate to study the cortical response to sensory stimuli, it is imperative to study the contributions in the cortical response to sensory stimuli due to nonlinearities in the system, which have never been systematically quantified.

Using multisine stimulation signals, which are designed by summing a specific set of sinusoidal signals [21], we can detect nonlinearities in the cortical response at frequencies which are not present in the stimulation signal. The goal of this paper is to quantify the nonlinear contributions to the cortical response obtained from continuous sensory stimulation. To the best of our knowledge, the cortical response obtained from continuous joint manipulation has only been investigated in one previous study by Campfens, et al. [18], however they analyzed the response only at the excited frequencies.

A robotic manipulator was used to apply continuous mechanical manipulation of the wrist joint and the response of the sensorimotor system was analyzed at three levels: mechanical response (joint angle and torque), muscle activity (electromyogram, EMG), and cortical activity (EEG). The nonlinear contributions are quantified in passive and active tasks, where the active tasks are performed to evoke and analyze EMG responses.

\section{MATERIALS AND METHODS}

\section{A. Subjects}

Eleven right-handed healthy volunteers ( 5 men, 6 women; age 22-25 years) with no self-reported history of neurological

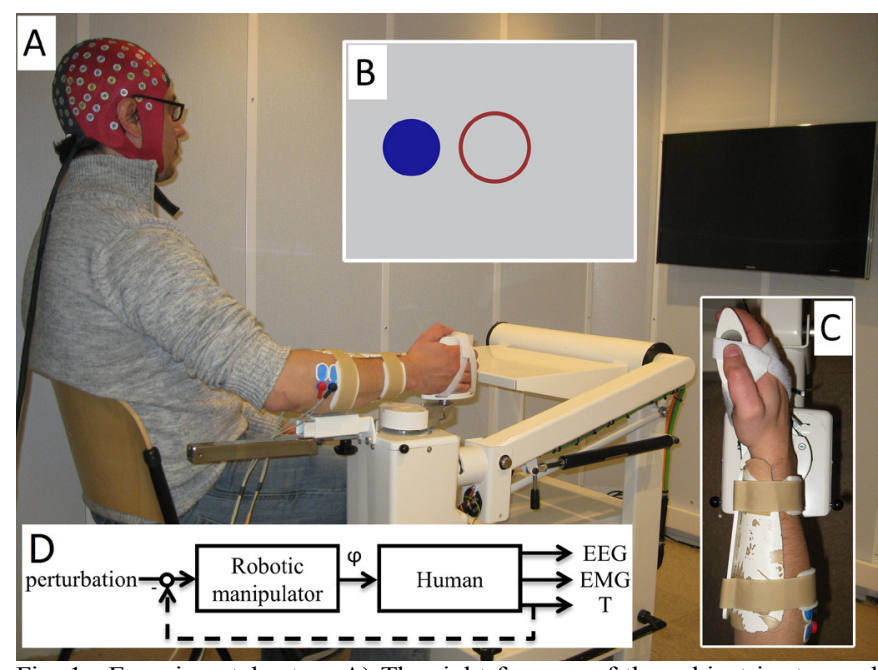

Fig. 1. Experimental setup. A) The right forearm of the subject is strapped into an armrest and the right hand is strapped to the handle, requiring no hand force to hold the handle. B) Visual feedback (the target (red circle) is static and always visible during a task, the blue circle is only visible during the active task and indicates the position of the handle and the task is to keep the blue circle in the red circle). C) Close up of the hand in the robotic manipulator. D) Block scheme depicting the robotic manipulator and the human. The perturbation signal (angle or torque) is applied to the human by the robotic manipulator, which will present the human with a certain angle $(\varphi)$. In case of the torque perturbation the torque on the handle $(\mathrm{T})$ is fed back to the robotic manipulator (dashed line). The robotic manipulator ensures the angle is set such that the torque on the handle (T) matches the perturbation signal.

disorders participated in this study. Subjects were all right handed (laterality index greater or equal to 80 according to the Edinburg Handedness Inventory [22]). The study was approved by the local research ethics committee. All subjects gave written informed consent prior to participation. Subjects were well rested and refrained from alcohol and drug intake 12 hours before the experiment.

\section{B. Experimental setup}

A one degree-of-freedom robotic wrist manipulator (Wristalyzer by MOOG Inc, Nieuw-Vennep, The Netherlands) applied the stimulation as angular or torque perturbations to the right wrist of the subjects (see Fig. 1A). The handle was adjusted so that the axis of rotation of the wrist was aligned with the axis of rotation of the manipulator (see Fig. 1C). The neutral angle was defined as the angle of the wrist when fully relaxed, resulting in a slight flexion posture. A screen placed at $1.5 \mathrm{~m}$ from the subject presented a target and task relevant feedback (see Fig. 1A\&B). The subjects were instructed to gaze at the center of the screen throughout the experiment to minimize head and eye movements. All recordings were performed in a slightly dimmed soundproof cabin.

Fig. 1D illustrates the closed-loop configuration of the robotic manipulator and the human, and indicates the recorded signals. All signals were sampled at $2048 \mathrm{~Hz}$ (136 channel Refa by TMSi, Oldenzaal, The Netherlands), ensuring synchronicity between all signals. Scalp potentials were measured using a cap with $126 \mathrm{Ag} / \mathrm{AgCl}$ electrodes (WaveGuard by ANT, Enschede, The Netherlands). The electrodes were arranged according to the $10-5$ system [23]. 
The mastoid electrodes on the cap were left unconnected. The subject ground electrode was connected to the left mastoid $(\mathrm{Ag} / \mathrm{AgCl}$ electrode, Blue Sensor $\mathrm{N}$ by Ambu, Ballerup, Denmark). Muscle activity was measured from the flexor carpi radialis $\left(\mathrm{EMG}_{\mathrm{F}}\right)$ and extensor carpi radialis $\left(\mathrm{EMG}_{\mathrm{E}}\right)$ using electrode pairs (Blue Sensor N by Ambu) attached to the skin and placed over the muscle belly with an electrode distance of $10 \mathrm{~mm}$. Handle angle and applied torque were measured from analog output signals of the wrist manipulator and were galvanic isolated from the amplifier using optical isolation amplifiers (TMSi, Oldenzaal, The Netherlands).

\section{Tasks}

Each subject performed two tasks: an active task and a passive task. In the passive task the subject was instructed to relax and to ignore the angular perturbations imposed by the manipulator. During the passive task the screen only presented the target and no feedback was given. The intensity of the angular perturbation was set such that the rotation of the handle had a root mean square $(\mathrm{rms})$ of $0.02 \mathrm{rad}(\approx 1.1 \mathrm{deg})$. Previous studies on the wrist joint applied perturbations in a similar range [18, 24].In the active task the subject was instructed to put effort in maintaining the wrist in the neutral angle while the manipulator imposed torque perturbations. During the active task the feedback screen presented the angle of the handle (see Fig. 1B), which was low-pass filtered online $\left(0.5 \mathrm{~Hz}, 2^{\text {nd }}\right.$ order Butterworth) to avoid rapid eye movements and to prevent the frequencies in the perturbation signal from stimulating the visual system. The intensity of the torque perturbations during the active task was iteratively set such that the rotation of the handle had an rms of around $0.02 \mathrm{rad}$. By studying the system for both tasks around the same operating point and applying small rotations allows for comparison between the tasks and facilitate linearization. Prior to the experiment subjects were required to practice the tasks.

\section{Perturbation signal selection}

The type of signal used to provide continuous manipulation of the wrist was a random phase multisine signal, which is a periodic signal consisting of several sinusoids summed together [21] as in:

$$
r(t)=\sum_{k=1}^{N} A_{k} \cos \left(2 \pi f_{0} k t+\phi_{k}\right),
$$

where:

- $k$ is the frequency line (integer number), which corresponds to the Fourier coefficients $(k=0$ is the DC coefficient and is omitted to obtain a zero-mean signal)

- $A_{k}$ is the amplitude at frequency line $k$ which can be zero or nonzero. Frequencies where the amplitude is nonzero compose the set of excited (i.e. stimulated) frequencies $\left\{f_{e x}\right\}$

- $f_{0}$ is the frequency resolution in hertz, defined by period length $T$ in seconds $\left(f_{0}=1 / T\right)$
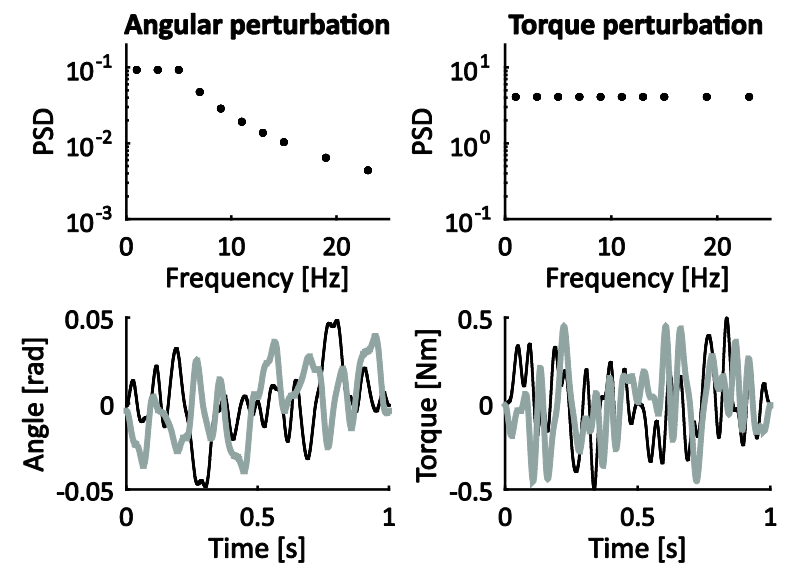

Fig. 2. Perturbation signals. Top graphs: power spectral density of the perturbation signals. Bottom graphs: a time domain representation of two out of seven realizations (thin black and thick gray) for both types of perturbation signal. The two realizations have identical frequency content and power however the phases are randomly distributed for each of the realizations resulting in different time courses of the signals (maximum correlation for any time shift for the two shown angular perturbation signals was 0.61 and for the torque perturbation signals 0.57 ).

- $\quad \phi_{k}$ is the random phase at frequency line $k$ which is taken from a uniform distribution

- $\quad N$ is the number of samples in $T$ which is defined by the sampling frequency

- $t$ is the time vector

Multisine signals allow for broadband excitation and system identification over a desired frequency range, and have several advantages in system identification over random perturbation signals such as (white) noise. Firstly, multisine signals allow concentrating signal power in a limited number of frequencies, which increases the input signal-to-noise ratio (SNR) at those frequencies while maintaining the same stimulation amplitude. Secondly, the noise levels can be quantified and reduced by recording multiple periods. Thirdly, multisine signals allow for leakage-free analysis due to their periodicity. Finally, by proper signal and experiment design a multisine perturbation signal allows for the detection and quantification of nonlinear distortions.

There exists a large class of nonlinear systems which, when excited with a periodic input signal, will generate a periodic response with the same period as the input. This class includes polynomials, saturations and rectifiers amongst many other systems. When repeatedly perturbing such a system with a multisine signal, the system will be excited in the same way and will therefore generate the same output [21]. The presence of nonlinear distortions generated by these systems can be revealed by using different realizations of a multisine signal, that have different phases but the same excited frequencies and amplitudes per frequency. As for a nonlinear system the superposition principle does not hold, perturbing the system using a different multisine realization (i.e. different time course) will excite the nonlinear system in a different way. This property will be exploited in the analysis by calculating to what extent a nonparametric linear model will be able to describe the input-output relation regarding all different realizations, which should be high for a linear system.

When using a multisine signal where only odd frequency 
lines are excited (e.g. $k=1,3,5)$ we can, besides quantifying the nonlinear contributions, also further describe the type of nonlinear behavior which will be helpful in a subsequent (non)parametric nonlinear modelling step. A linear system will show a response only at the excited frequencies. Nonlinear systems will show a response at unexcited frequencies, which can be harmonics of the excited frequencies (e.g. $2 f_{e x_{1}}$ ) or intermodulation products (e.g. $\left.f_{e x_{1}}+f_{e x_{2}}\right)$. Nonlinear systems can have an odd or even behavior, or show both behaviors at the same time. When an odd nonlinear system (i.e. $y(u)=-y(-u)$ ) is excited at an odd frequency line, the response will only contain power at harmonic odd frequency lines. When an even nonlinear function (i.e. $y(u)=y(-u)$ ) is excited at an odd frequency line, the response will only contain power at harmonic even frequency lines. More generally, when perturbing a system with a signal containing only odd frequency lines, any power that is present in the (noise free) output signal at the even frequency lines must be due to nonlinear distortions generated by an even or even and odd nonlinear function. Additionally, exciting only odd frequency lines ensures there is no disturbing effect of even nonlinear distortions on the excited frequency lines. This characteristic allows for differentiation between even and odd nonlinear distortions, while maintaining the ability to perform system identification over the chosen (odd) frequency range of interest.

\section{E. Perturbation signal design}

Multisine perturbation signals with a period of $1 \mathrm{~s}$ were designed, resulting in a frequency resolution of $1 \mathrm{~Hz}$. The excited odd frequency lines are 1, 3, 5, 7, 9, 11, 13, 15, 19, 23 $\mathrm{Hz}$, resulting in a total of 10 excited frequencies. As mentioned above, leaving the even frequency lines unexcited allows for the detection of even nonlinear distortions. Some odd frequency lines $(17,21,25 \mathrm{~Hz}$ and higher) are not excited to allow for detection of odd nonlinear distortions. The selected excited frequency lines are a trade-off between frequency resolution and the ability to detect odd nonlinear distortions. The dynamics of the wrist are observable within this frequency range [25]. Seven different realizations of a random phase multisine were applied, as at least seven realization are needed to preserve the properties of the maximum likelihood estimator (such as consistency) in possible future parametric modelling steps [26].

All perturbation signals were generated offline and the same set of signals was used for all subjects (see Fig. 2). The angular perturbation signal was designed to have equal power on the first three excited frequencies and a decreasing power for the higher frequencies $(-20 \mathrm{~dB} /$ decade slope), which is a tradeoff between reduced predictability of the signal (to prevent anticipation) and capabilities of the robotic manipulator. The torque perturbation signal was designed to have equal power on all excited frequencies. For each perturbation signal seven realizations of a random phase multisine were generated. To ensure these realizations are actually different and therefor excite the (non)linear system in a different way, the correlation amongst these seven signals was controlled. This was achieved by generating 25000 random-phase multisine signals and removing $10 \%$ of the signals having the highest peak-to-rms ratio (crest factor) and another $10 \%$ having the least normal distribution (chi-square test). Out of the remaining 20000 signals, seven realizations were obtained with a correlation coefficient of less than 0.65 amongst each other for any time shift, which was found to be the lowest achievable number with the used signal properties.

\section{F. Experimental protocol}

To prevent fatigue the experiment was partitioned in trials of $36 \mathrm{~s}$. There was a break between trials of at least $10 \mathrm{~s}$ or longer if requested by the subject. The active and passive trials were alternately presented to the subject. To avoid habituation to the signals each trial consisted of three randomly selected multisine realizations, which were repeated several times and smoothly merged. A smooth transition from one multisine realization to another was achieved by shifting all seven multisine realizations to have an amplitude and velocity close to zero at the beginning (and therefore also end) of its period. The transition between two concatenated realizations was further smoothed by interpolating between the last sample of the first realization up to the 50th sample $(\sim 25 \mathrm{~ms})$ of the second realization using piecewise cubic spline interpolation. Periods containing the transition between two realizations were removed. Additionally, the first four periods of each trial were removed from the analysis to account for transient effects, resulting in a total of ten useful periods for each of the three multisine realizations in the trial (see Fig. 3). A total of 49 trials (i.e. 1470 useful periods) was recorded per task, consisting of 210 periods $(\mathrm{P}=210)$ for each of the seven realizations $(\mathrm{M}=7)$. Including mandatory breaks, this protocol resulted in a minimal recording time of 76 minutes for each subject.

\section{G. Pre-processing}

Data processing was performed using FieldTrip [27] and MATLAB 8.1 (The Mathworks, Inc., Natick, MA, USA). Line noise $(50 \mathrm{~Hz}$ and its harmonics) was removed using the discrete Fourier transform as implemented in FieldTrip. The EMG signals were high-pass filtered in two directions $(25 \mathrm{~Hz}$, $4^{\text {th }}$ order Butterworth) to remove motion artifacts introduced

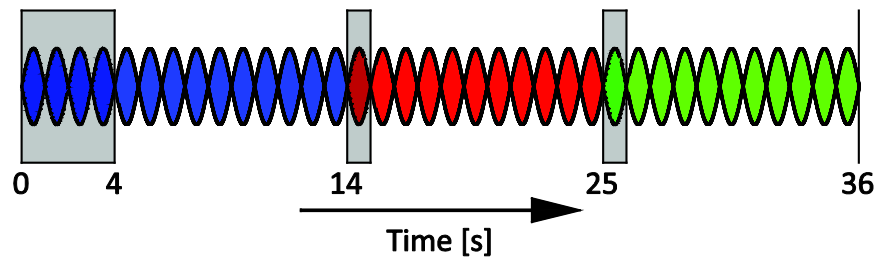

Fig. 3. Schematic representation of composition of one 36s trial. Each lobe represents one 1s period of the perturbation signal and the three different colors represent different multisine realizations. Highlighted periods are excluded from analysis, leaving ten periods per realization in each trial for analysis. 
by the robotic manipulator, and were subsequently rectified. The mean of the rectified EMG signal was removed as we are interested in the response of the muscle to the perturbation (i.e. reflexive contributions) and less in tonic activation and the generated torque [8]. The EEG signals were referenced to the common average and high-pass filtered in two directions ( $1 \mathrm{~Hz}, 4^{\text {th }}$ order Butterworth). No artifact rejection was applied.

\section{H. Data analysis}

Since periodic perturbation signals were used, all recorded signals were organized in $\mathrm{P}$ periods of $\mathrm{M}$ realizations ( $\left.x^{[m, p]}(t)\right)$. Consequently any part of the response that is not periodic with the same period as the perturbation signal will be regarded as noise.

1) Quantifying the relative power of the nonlinear distortions

The frequency domain representation of the recorded signals was obtained by applying the Fourier transform, resulting in $X^{[m, p]}(f)$. An estimate of the total power in each signal was obtained by averaging over periods (P) and thereby reducing the noise, calculating the power, averaging over realizations $(\mathrm{M})$ and summing over all frequencies $(\mathrm{F})$ :

$\hat{E}_{X, \text { total }}=\sum_{f=1}^{F} \frac{1}{M} \sum_{m=1}^{M}\left|\frac{1}{P} \sum_{p=1}^{P} X^{[m, p]}(f)\right|^{2}$.

The power in the excited $\left(\hat{E}_{X, e x}\right)$, unexcited odd $\left(\hat{E}_{X \text {,unex,odd }}\right)$, and unexcited even $\left(\hat{E}_{X, \text { unex,even }}\right)$ frequencies can be determined by summing over a specific set of frequencies in (2), where:

$$
\hat{E}_{X, \text { total }}=\hat{E}_{X, e x}+\hat{E}_{X, \text { unex,odd }}+\hat{E}_{X, \text { unex, even }} .
$$

The relative power in these frequencies can be estimated by dividing the power in excited, unexcited odd, or unexcited even frequencies by the total power.

2) Noise-to-signal ratio

An estimate of the noise level in the recorded signals was made by calculating the variance over periods $(\mathrm{P})$, averaging this variance over all realizations $(\mathrm{M})$, and summing over all frequencies $(\mathrm{F})$ :

$$
\hat{\sigma}_{X}^{2}=\sum_{f=1}^{F} \frac{1}{M} \sum_{m=1}^{M} \frac{1}{P-1} \sum_{p=1}^{P}\left|X^{[m, p]}(f)-\frac{1}{P} \sum_{p=1}^{P} X^{[m, p]}(f)\right|^{2} .
$$

The noise-to-signal ratio (NSR) for each recorded signal was obtained by dividing the estimate of the noise level by the estimate of the power in the signal, and is used to select the electrode showing the strongest response relative to the noise level:

$$
N S R=\frac{\hat{\sigma}_{X}^{2}}{\hat{E}_{X, \text { total }}} .
$$

The sample noise level on the averaged data (i.e. standard error of the mean) compared to the sample mean is referred to as the $\mathrm{NSR}_{\text {scaled, }}$ and is an estimate of the amount of noise still present in the averaged data:

$N S R_{\text {scaled }}=\frac{N S R}{P}$.

For each subject the EEG signal at the electrodes with the lowest NSR in the passive and active task were used for subsequent analysis and were named $\mathrm{EEG}_{\mathrm{P}}$ and $\mathrm{EEG}_{\mathrm{A}}$ respectively.

\section{3) Determining the best linear approximation}

System identification was used to determine how much of the recorded signals can be described by a nonparametric linear model. We obtained such a linear model by nonparametric estimation of the frequency response function (FRF). . In agreement with the system depicted in Fig. 1D, an FRF of the human was estimated with the perturbation signal as external reference signal (R), angle $\varphi$ as input $(U)$ and the torque, EMG and EEG signals as output signal $(Y)$. The inputoutput relations are given by the measured FRFs $G_{T \varphi}, G_{E M G_{F} \varphi}$ , $G_{E M G_{E} \varphi}$ and $G_{E E G_{A} \varphi}$.

Each FRF $G(f)$ consists of three parts [21]:

$$
G(f)=G_{B L A}(f)+G_{S N L}(f)+G_{\text {noise }}(f) \text {. }
$$

Here $G_{B L A}$ is the best linear approximation (BLA) of the (non)linear system under study, $G_{S N L}$ represents the stochastic nonlinear distortions, and $G_{\text {noise }}$ the errors due to the presence of noise. $G_{\text {noise }}$ is assumed to be uncorrelated with the reference signal and to have zero mean. In case of a random reference signal $G_{S N L}$ will appear as uncorrelated zero-mean noise, however not in case of a periodic deterministic reference signal such as the used multisine signals. This implicates that $G_{\text {noise }}$ will be different for each period in each realization whereas $G_{S N L}$ will be the same in each period in a realization, but will differ over realizations. $G_{B L A}$ was estimated for each of the four FRFs using a closed loop estimator:

$\hat{G}_{B L A}\left(f_{e x}\right)=\frac{\hat{S}_{\hat{Y} R}\left(f_{e x}\right)}{\hat{S}_{\hat{U} R}\left(f_{e x}\right)}$.

Here $\hat{S}(f)$ is the estimated cross-spectral density, averaged over periods and realizations, which reduces the contributions of noise and stochastic nonlinear distortions in the final estimate. The cross-spectral density was calculated between the perturbation signal $R$ and input $U(\varphi)$ and output $\mathrm{Y}$ (torque, EMG and EEG) at the excited frequencies. A detailed overview of the equations used to obtain an estimate of $G_{B L A}$ and its noise variance can be found in appendix A.

To quantify how well the nonparametric transfer function $G_{B L A}$ describes the measured data, we used the variance accounted for (VAF). The model output $Y_{\text {mod }}$ was determined using: 


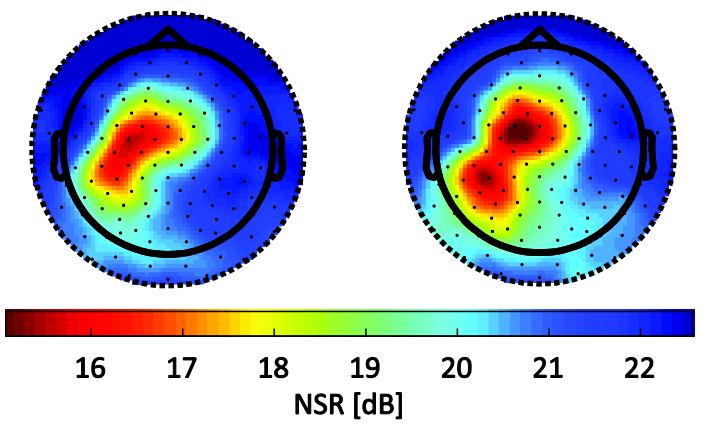

Fig. 4. NSR per electrode for passive task (left) and active task (right) averaged over all subjects. Dots indicate electrode locations. The lowest NSR is found over the contralateral sensorimotor areas.

$Y_{\bmod }^{[m]}\left(f_{e x}\right)=\frac{1}{P} \sum_{p=1}^{P} U^{[m, p]}\left(f_{e x}\right) \hat{G}_{B L A}\left(f_{e x}\right) ，$

and converted to the time domain, using the inverse Fourier transform:

$y_{\text {mod }}^{[m]}(t)=\mathcal{F}^{-1}\left(Y_{\text {mod }}^{[m]}\left(f_{e x}\right)\right)$

The model output $y_{\bmod , c}^{[m]}$ and the average recorded output $\hat{y}^{[m]}$ of the seven different realizations were concatenated into $y_{\bmod , c}$ and $\hat{y}_{c}$ respectively. The VAF for each recorded output signal (torque, $\mathrm{EMG}_{\mathrm{F}}, \mathrm{EMG}_{\mathrm{E}}$ and $\mathrm{EEG}_{\mathrm{P}}$ or $\mathrm{EEG}_{\mathrm{A}}$ ) was obtained using:

$V A F=\left(1-\frac{\operatorname{var}\left(\hat{y}_{c}-y_{\text {mod }, c}\right)}{\operatorname{var}\left(\hat{y}_{c}\right)}\right) \cdot 100 \%$

\section{RESULTS}

This section presents the averaged NSR, power distribution over frequency groups and VAFs as well as individual results of a representative subject. In both the passive and active task we were able to quantify the nonlinear contributions to the mechanical and EEG data. Additionally, the active task also allowed us to study the nonlinear contributions to the EMG data. One out of the eleven subjects was not included in the analysis for not being able to successfully complete the active task. In one other subject one electrode (FT9) was removed from the analysis because it was accidentally disconnected during the experiment due to the improper placement of glasses.

\section{A. Noise-to-signal ratio}

Fig. 4 illustrates the averaged NSR in the passive and active tasks. The lowest NSR for both tasks appears around the contralateral sensorimotor areas. An decreased NSR indicates there is a periodic response in the brain due to the external perturbation signal, which is reproducible over trials.

For all subjects the electrode which had the lowest NSR was found on the contralateral hemisphere, close to the

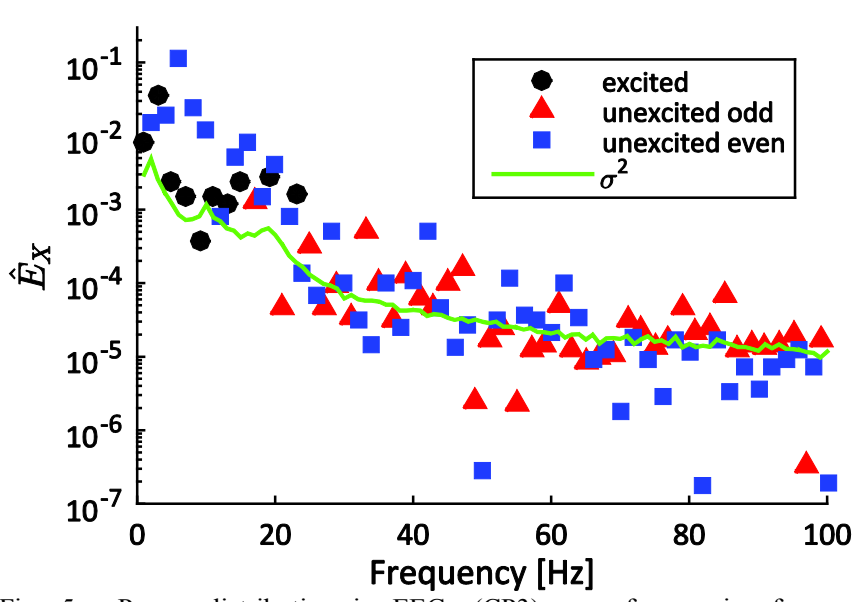

Fig. 5. Power distribution in EEG $(\mathrm{CP} 3)$ over frequencies for one realization of the passive task for a representative subject. Results are averaged over $\mathrm{P}=210$ periods. Black dots represent the excited frequencies, red triangles represent the unexcited odd frequencies and the blue squares represent the unexcited even frequencies. The (scaled) noise level is indicated by the green line.

sensorimotor areas in the passive $\left(\mathrm{EEG}_{\mathrm{p}}\right.$ : $3 \mathrm{x} \mathrm{FCC} 3 \mathrm{~h}, 2 \mathrm{x} \mathrm{CP} 3$, 2x FCC1h, 1x C3, 1x CCP3h and 1x FC1) and active task $\left(\mathrm{EEG}_{\mathrm{A}}\right.$ : 4x CP3, 2x FC1, 2x FCC1h, 1x CCP3h and 1x FCC3h).

The first two columns in Table I show the NSR and noise levels in the recorded signals (angle, torque, EMG and EEG) averaged across persons. The NSR of the recorded angle $(\varphi)$ is lower for the passive task because the robotic manipulator directly controls the angle, while the angle in the active task is the result of the human responding to torque perturbations.

The noise level in the EMG signals for the passive task indicates there is not a consistent EMG response to the perturbations, as expected. The noise levels in the EMG signals for the active task and the $E_{E G_{P}}$ and $E_{E G}$ signals indicate that there is still some noise present after averaging over 210 periods, thereby limiting the maximal attainable VAF. However, these numbers also indicate that over $80 \%$ of the recorded physiological data can be described when a proper model is used.

\section{B. Power of nonlinear distortions}

Fig. 5 shows the power distribution over frequencies for $E G_{P}$ for one realization of a representative subject. We can observe that most power is in the unexcited even frequencies, while the power in the excited and unexcited odd frequencies is much lower. The noise level clearly shows two peaks around 10 and $20 \mathrm{~Hz}$; most likely representing the intrinsic alpha and beta band rhythms. It can also be seen that close to $100 \mathrm{~Hz}$ the power becomes small and the NSR increases. The effect of the line noise filter is clearly visible at $50 \mathrm{~Hz}$ and 100 Hz. The averaged power distribution, NSR, and noise levels for the signals of interest can be found in Table I. The power distribution for the EMG signals in the passive task is not shown, due to the inherently high NSR. Fig. 5 as well as Table I indicate that most of the power in the EEG signal is in the unexcited even frequencies. 


\section{Frequency response functions}

The four FRFs for a representative subject are shown in Fig. 6. The mechanical admittance $\left(G_{\varphi T}\right)$ behaves like a second order system for both tasks, which was previously established [28]. As expected, the mechanical admittance is higher for the passive task compared to the active task, where the instruction was to maintain the angle by resisting the perturbation, i.e., to lower the admittance. This increased stiffness can be generated by co-contracted muscles as well as reflexive activity (primarily from the spinal reflex loop). Due to the increased stiffness in the active task the natural frequency of the wrist also increases from approximately $3 \mathrm{~Hz}$ to $5 \mathrm{~Hz}$, which can be observed in the shifted resonance peak in the gain plot. The high frequency response, which is governed by the inertia of the wrist, is similar in both tasks. This result was expected as the inertia does not vary over tasks.

The reflexive impedance $\left(G_{E M G \varphi}\right)$ for the passive task is much lower than for the active task and is of the same order of magnitude as the noise level, also indicating a high NSR. The reflexive activity was minimal in the passive task as compared to the active task. The reflexive impedance for the active task shows similar behavior between flexor and extensor muscles, except for the phase being 180 degrees shifted. This corresponds to the unidirectional nature of the muscles, which are only able to actively contract. The increasing phase lag at the higher frequencies is caused by the neural time delay in the reflex loop.

The FRF for $G_{E E G \varphi}$ indicates that the linear transfer function is of the same order of magnitude as the noise level. Together with the non-smooth and erratic behavior of both gain and phase, this indicates a low quality nonparametric linear model.

\section{Fitting the best nonparametric linear model}

We used the best linear approximation as a nonparametric model and obtained the VAF for each of the four input-output relations (see Table II). For the passive task the VAF for the relation between the angle $\varphi$ and the EMG signals is not calculated, since there is no consistent EMG response to the perturbation in this task. An example of the model fit in the time domain for the active task for one representative subject can be found in Fig. 7.

The average VAF for $G_{\varphi T}$ (i.e. mechanical admittance) is around $99 \%$ for both the passive task and the active task, indicating the relation between angle and torque is well described by a nonparametric linear model. Due to the high number of recorded periods and subsequent low noise level in this study, the VAF was high compared to other studies were the mechanical admittance was modelled from much less recorded periods. In previous studies on the wrist and other joints $[8,19,25,29]$ a VAF between $80 \%$ and $95 \%$ was obtained when using a parametric linear model to describe the relation between angle and torque. The averaged VAF obtained when modelling the EMG signal was around $70 \%$ for both muscles, which is slightly higher than the same studies mentioned before where a VAF of $40 \%$ to $60 \%$ was common when modelling the EMG signals with a parametric linear model. Besides the low noise level, the high flexibility of the nonparametric models used in this study compared to parametric models resulted in a higher VAF. Noteworthy, the relation between the wrist angle and the measured EEG signal is poorly captured by the nonparametric linear model. The averaged VAF is around $10 \%$ for both the passive task and active task, thus a linear system description is not appropriate to describe the response in the EEG evoked by mechanical manipulation of the wrist.

\section{DISCUSSION}

Mechanical manipulation of the wrist using multisine signals elicits a periodic response in the EEG, which is shown to be highly nonlinear. Linear system identification techniques were employed and indicate that the wrist torque and EMG response to small changes in wrist angle can be explained for $99 \%$ and $70 \%$ respectively using a nonparametric linear model. Following the same approach, the response in the EEG could only be explained for $10 \%$ with linear methods. Moreover, the power in the cortical response at the unexcited frequencies (i.e. due to nonlinear behavior) is over $80 \%$. Similar results were obtained for all subjects. Thus, we conclude that a linear description of the relation between stimulus and response in the EEG is inappropriate and a nonlinear description is required.
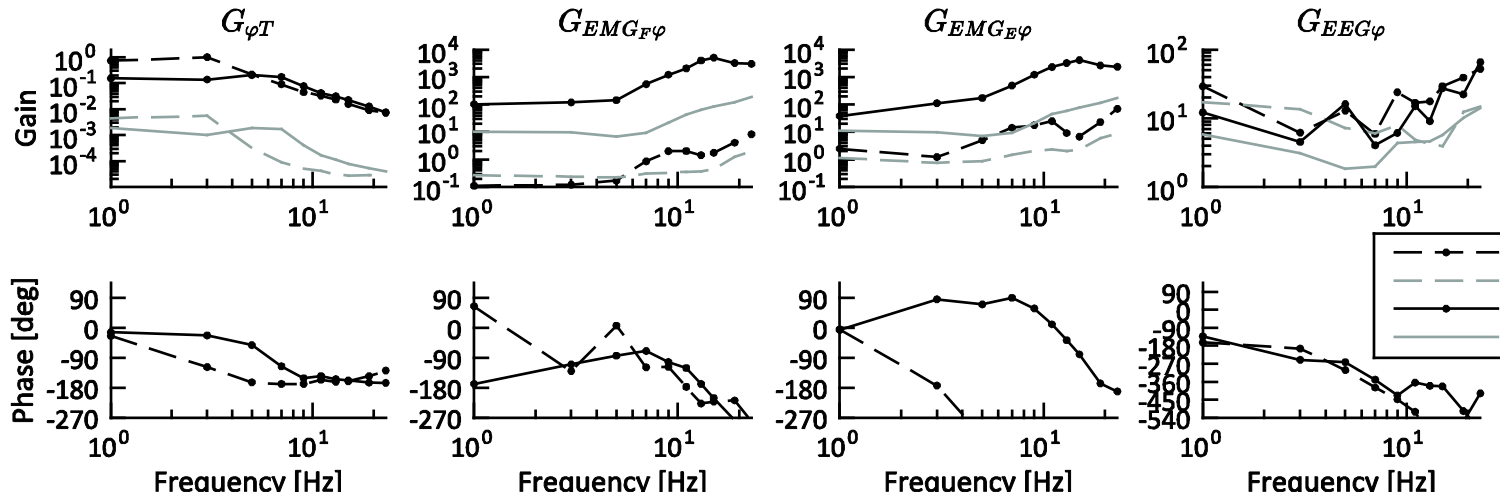

Frequency $[\mathrm{Hz}]$

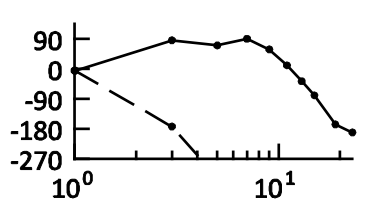

Frequency $[\mathrm{Hz}]$

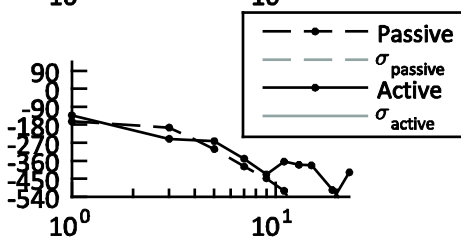

Frequency $[\mathrm{Hz}]$

Fig. 6. Frequency response functions (gain and phase) for a representative subject. The units for $G_{\varphi T}$ are $[\mathrm{rad} / \mathrm{Nm}]$ and for the other FRF's [ $\left.\mu \mathrm{V} / \mathrm{rad}\right]$. Black lines with markers indicate the FRF at the excited frequencies and gray lines the scaled noise level (see Appendix A for equations). Solid and dashed lines represent the active task and passive task respectively. The transfer function $G_{\varphi T}$ is presented as an admittance to correspond to existing literature $\left(G_{\varphi T}=G_{T \varphi}{ }^{-1}\right)$. 

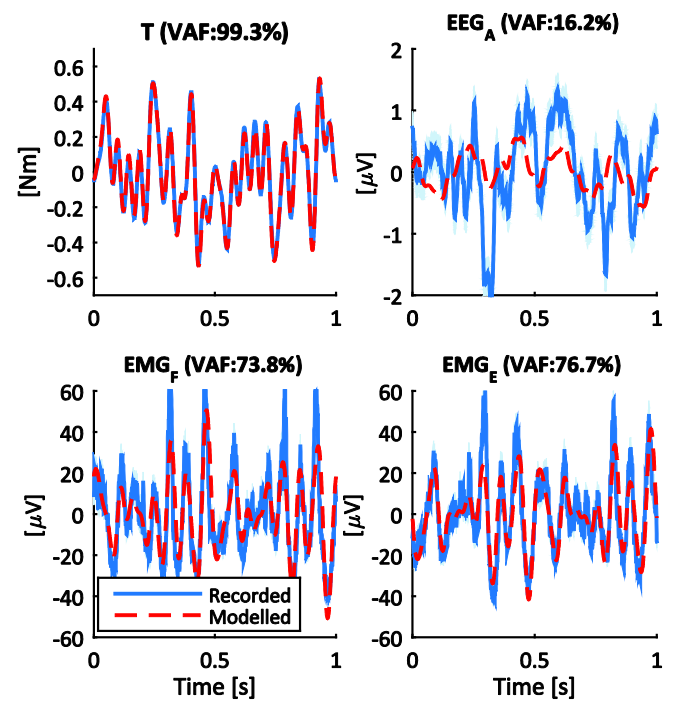

Fig. 7. Time domain fit of nonparametric linear model (dashed red line) on top of the averaged recorded output (blue line, light blue area represents the averaged response \pm the noise level) for one realization of the active task for a representative subject.

\section{A. Quantification of the nonlinear contributions}

The use of multisine perturbation signals with power concentrated in a limited number of odd frequency lines, allowed assessment of any power transferred from excited to unexcited frequency lines, which is caused by nonlinear behavior. The used method can detect nonlinear distortions which are periodic with the same period as the perturbation signal (e.g., polynomials, saturations and rectifiers). Nonlinear distortions generated by nonlinearities such as chaos and bifurcations, as well as distortions due to time-variant behavior, can therefore not be detected and will increase the noise level. In a previous study using a similar setup, we demonstrated there was no substantial time-variant behavior in the EEG signals evoked by wrist manipulation [30].

The power in the excited frequencies should not be regarded as 'the linear part of the response' [14] because odd nonlinear functions can very well affect the signal at the odd excited frequencies. Therefore analyzing the response only at the fundamental (excited) frequencies must be differentiated from studying the linear part of the response. By estimating a

TABLE I

AVERAGE NOISE-TO-SIGNAL RATIO AND

RELATIVE POWER DISTRIBUTION OVER FREQUENCY GROUPS

\begin{tabular}{cccccc}
\multicolumn{7}{c}{ RELATIVE POWER DISTRIBUTION OVER FREQUENCY GROUPS } \\
\hline \hline & $\begin{array}{c}\text { NSR } \\
{[\mathrm{dB}]}\end{array}$ & $\begin{array}{c}\mathrm{NSR}_{\text {scaled }} \\
{[\%]}\end{array}$ & $\begin{array}{c}\mathrm{E}_{\mathrm{ex}} \\
{[\%]}\end{array}$ & $\begin{array}{c}\mathrm{E}_{\text {unex-odd }} \\
{[\%]}\end{array}$ & $\begin{array}{c}\mathrm{E}_{\text {unex-even }} \\
{[\%]}\end{array}$ \\
& \multicolumn{5}{c}{ Passive task mean (sd) } \\
\hline $\boldsymbol{\varphi}$ & -30.9 & $0.0(0.0)$ & $100(0.0)$ & $0.0(0.0)$ & $0.0(0.0)$ \\
$\mathbf{T}$ & -18.8 & $0.0(0.0)$ & $99.6(0.2)$ & $0.2(0.1)$ & $0.2(0.1)$ \\
$\mathbf{E M G}_{\mathbf{F}}$ & 20.0 & $58.9(24.4)$ & - & - & - \\
$\mathbf{E M G}_{\mathbf{E}}$ & 19.9 & $55.9(24.8)$ & - & - & - \\
$\mathbf{E E G}_{\mathbf{P}}$ & 14.8 & $17.1(7.2)$ & $18.6(5.7)$ & $6.0(2.1)$ & $75.4(5.6)$ \\
& \multicolumn{7}{c}{ Active task mean (sd) } \\
$\mathbf{\varphi}$ & -8.9 & $0.1(0.0)$ & $99.6(0.2)$ & $0.0(0.0)$ & $0.4(0.2)$ \\
$\mathbf{T}$ & -19.6 & $0.0(0.0)$ & $99.4(0.3)$ & $0.1(0.0)$ & $0.5(0.3)$ \\
$\mathbf{E M G}_{\mathbf{F}}$ & 10.8 & $7.4(3.9)$ & $77.5(4.9)$ & $5.6(1.6)$ & $16.8(4.3)$ \\
$\mathbf{E M G}_{\mathbf{E}}$ & 12.0 & $10.7(7.8)$ & $76.4(7.4)$ & $8.8(3.4)$ & $14.8(4.8)$ \\
$\mathbf{E E G}_{\mathbf{A}}$ & 14.2 & $13.2(3.6)$ & $16.8(3.3)$ & $2.6(0.9)$ & $80.7(4.1)$ \\
\hline \hline
\end{tabular}

(linear) FRF at the excited frequencies and by calculating the VAF, we can assess how well such a linear nonparametric model can describe the input-output relations.

Our results show that for small excursions around an operating point as used in this study the wrist dynamics are mainly linear, as almost $100 \%$ of the power in the recorded angle and torque signals is present in the excited frequencies and the VAF when using a nonparametric linear model is over $99 \%$.

For the EMG recordings in the active task the power in the excited frequencies is around $76 \%$. There is clear evidence of nonlinear distortions in the EMG signals, which are possibly introduced by the unidirectional nature of a muscle and stretch reflex. Previous studies on reflex dynamics indeed showed the muscle spindle and reflex loop behaving as a half-wave rectifier [31]. A linear model can still describe the EMG response for approximately $70 \%$. Even though the reflexive impedance is nonlinear, paradoxically the mechanical admittance behaves linear. The relation between joint angle and muscle EMG is nonlinear, however the flexor and extensor muscles act as two opposing half-wave rectifiers, therewith linearizing the net reflexive behavior. Here, linearization is facilitated by the small amplitude of the perturbations and the neutral wrist angle, which allows for similar contribution from flexor and extensor muscles.

In contrast to the mechanical and EMG signals, the excited frequencies for the EEG signal account for only $17 \%$ of the total signal power, indicating the EEG signal is dominated by nonlinear contributions. The relation between input and output therefore cannot be described by an FRF at the excited frequencies, since it only takes a small portion of the output into account and therefore results in a low VAF $(\sim 10 \%)$. Most power in the EEG signal, over $75 \%$ for both the passive and active task, is in the even frequency lines, indicating the presence of a dominant even nonlinear function. Examples of an even nonlinear function are $y(u)=u^{2}, y(u)=u^{4}$ and $y(u)=a b s(u)$. Seiss et al. [32] and Campfens, et al. [33] showed that a stretch of respectively the finger and wrist resulted in a similar ERP for both flexion and extension direction, which also indicates an even nonlinear relation.

\section{B. Origin of nonlinear contributions}

The nonlinear behavior of the muscle spindles [34-36] is likely to add to the nonlinear contributions in the EMG

TABLE II

VAF FOR THE FOUR NONPARAMETRIC LINEAR MODELS

\begin{tabular}{ccc}
\hline \hline & $\begin{array}{c}\text { Passive task } \\
{[\%]}\end{array}$ & $\begin{array}{c}\text { Active task } \\
{[\%]}\end{array}$ \\
\hline$G_{T \varphi}$ & $99.5(0.2)$ & $99.0(0.3)$ \\
$G_{E M G_{F} \varphi}$ & - & $70.0(6.4)$ \\
$G_{E M G_{E} \varphi}$ & - & $68.0(8.1)$ \\
$G_{E E G \varphi}$ & $10.0(4.0)$ & $10.3(2.3)$ \\
\hline \hline
\end{tabular}


response. Their unidirectional sensitivity to velocity changes, together with their position in antagonistic muscles, could result in a similar neural signal for both flexion and extension. When using EEG to record these neural signals from the cortex, the distance between processing sites might be too small to be distinguishable, resulting in a lumped response of flexor and extensor muscle spindles. This response would, due to the similar signal for flexion and extension, result in an even nonlinear relation between stimulus and recorded EEG.

\section{Passive and active task}

Similar results for the EEG response are obtained for the passive and active task: the NSR as well as the distribution of power over frequency groups are of the same order of magnitude in both tasks. The NSR is slightly lower in the active task and low NSR is found in a larger region compared to the passive task. There are several possible explanations for these small changes. In the active task the muscles are generating force due to both co-contraction and reflexive activity, resulting in increased wrist torque. Compared to the passive task, this will result in changed muscle spindle sensitivity [37] and an increase in output of the Golgi tendon organ [38]. Changes in EEG could also be due to the involvement of additional brain regions in voluntary cocontraction during the active task (e.g., supplementary motor area, pre-motor cortex, posterior parietal cortex) [39].

\section{Implications}

When applying a mechanical (multi)sine stimulus signal to a linear system, the response will occur only at the exited frequencies. In a nonlinear system, the frequency domain analysis of the response must consider excited frequencies and their harmonics and intermodulation products. Taking all these components into account will elucidate which nonlinear model could appropriately describe the relationship. In this study we have shown that the larger part of the EEG response to small mechanical perturbations, and therefore most information, is found in the unexcited frequencies, indicating nonlinear behavior.

When applying transient sensory stimuli, the resulting average response is called the event-related potential (ERP). Information about the sensory system is derived from the timing of certain components in the ERP, for example the negative deflection $20 \mathrm{~ms}$ after electrical stimulation (N20) (see [40] for more details on somatosensory evoked potentials). Although the ERP technique is widely used, it often overlooks that neurophysiological systems exhibit nonlinear behavior. In a linear system the response scales proportionally with the stimulus amplitude and the shape of the response remains the same (e.g. doubling the input amplitude results in a doubled output amplitude), whereas in a nonlinear system both the shape and amplitude of the response will vary with applied stimulus amplitude. Several studies on mechanically somatosensory responses show that the shape of the ERP changes with the amplitude of the mechanical stimulus [41-44]. Due to this nonlinear behavior of the system (also shown in our results) the shape of the response and subsequently the timing of characteristic peaks will change with a change in amplitude of the sensory stimulus. Due to the nonlinear behavior of the system its characteristics cannot be fully captured by responses to a transient stimulus (ERP) or by responses to a continuous stimulus at only the excited frequency [e.g. 45, 46, 47].

Our results have shown that the relation between mechanical manipulation of the wrist joint and the response in the EEG cannot be described by a nonparametric linear model (VAF: $\sim 10 \%$ ), demonstrating nonlinear behavior. Consequently, a linear model or method will not be able to capture the relation between stimulus and response. An example is found in directed corticomuscular (linear) coherence, where poor linearization might contribute to inconsistently estimated time delays between cortex and muscle (e.g. [48]).

The observed nonlinear behavior is periodic with the same period as the perturbation signal. Even though there is still substantial noise left in the EEG signals after averaging, a perfect model should be able to describe over $80 \%$ of the relation between wrist movement and recorded EEG. By using nonlinear modelling techniques we should be able to provide a better description of the input-output relationship. There exists an infinite amount of nonlinear operators and nonlinear model structures and this study provides essential information on the nature of the nonlinearity in the system. The next step in this research will be to obtain a nonlinear model relating the imposed wrist movement to the recorded EEG signals, therewith improving the understanding of the human sensory system and ultimately providing insight in movement disorders.

Both ERP's and cortical responses to continuous stimulation have been obtained using other types of stimuli such as visual, auditory and electrical nerve stimulation. The cortical responses to these types of stimuli also shows higher harmonics of the stimulation frequency [49]. The nonlinear contributions to the response, when these stimuli are applied, can be quantified using the techniques described in this study.

\section{CONCLUSIONS}

- Multisine perturbation signals applied to the wrist elicit a periodic cortical response and allow assessment of nonlinear contributions to the response.

- When studied in a small range, wrist dynamics can be successfully linearized.

- The relationship between mechanical stimulus and cortical response is highly nonlinear. Over $80 \%$ of the cortical response is caused by nonlinear behavior of the system. We showed that a nonparametric linear model only explains $10 \%$ of the cortical response to mechanical joint manipulation.

- Event related potentials are insufficient to fully characterize the highly nonlinear relationship between mechanical stimulus and EEG response. 


\section{Appendix A}

The following equations allow for estimation of the sample mean and sample (co)variance for each recorded signal or signal combination [21]. $X(f)$ and $Z(f)$ refer to Fourier transformed recorded signals, which can be the same or different signals.

First the phase in the recorded signal is turned back by the phase in the perturbation signal (R) (12), which allows for averaging over the different realizations (14):

$$
\begin{aligned}
& X_{R}^{[m, p]}(f)=\frac{X^{[m, p]}(f)}{e^{j<R^{[m]}}(f)}, \\
& \hat{X}_{R}^{[m]}(f)=\frac{1}{P} \sum_{p=1}^{P} X_{R}^{[m, p]}(f), \\
& \hat{X}_{R}(f)=\frac{1}{M} \sum_{m=1}^{M} X_{R}^{[m]}(f) .
\end{aligned}
$$

The sample (co)variance for each recorded signal or signal combination is estimated in (15) and averaged over realizations in (16):

$$
\begin{aligned}
& \left.\hat{\sigma}_{X_{R} Z_{R}, n}^{[m]}(f)=\frac{1}{P(P-1)} \sum_{p=1}^{P}\left(\frac{\left(X_{R}^{[m, p]}(f)-\hat{X}_{R}^{[m]}(f)\right)}{Z_{R}^{[m, p]}(f)-\hat{Z}_{R}^{[m]}(f)}\right)\right) \\
& \hat{\sigma}_{X_{R} Z_{R}, n}^{2}(f)=\frac{1}{M^{2}} \sum_{m=1}^{M} \hat{\sigma}_{X_{R} Z_{R}, n}^{2[m]}(f)
\end{aligned}
$$

The FRF and its noise variance at the excited frequencies are obtained by inserting the recorded input and output signals of interest into equation A1-A5 and subsequently inserting the result in (17) and (18):

$$
\begin{aligned}
& \hat{G}_{B L A}\left(f_{e x}\right)=\frac{\hat{S}_{\hat{Y} R}\left(f_{e x}\right)}{\hat{S}_{\hat{U} R}\left(f_{e x}\right)}=\frac{\hat{Y}_{R}\left(f_{e x}\right)}{\hat{U}_{R}\left(f_{e x}\right)}, \\
& \hat{\sigma}_{N_{G}}^{2}\left(f_{e x}\right)=\left|\hat{G}_{B L A}\left(f_{e x}\right)\right|^{2}\left(\begin{array}{l}
\frac{\hat{\sigma}_{Y_{R}, n}^{2}\left(f_{e x}\right)}{\left|\hat{Y}_{R}\left(f_{e x}\right)\right|^{2}}+\frac{\hat{\sigma}_{U_{R}, n}^{2}\left(f_{e x}\right)}{\left|\hat{U}_{R}\left(f_{e x}\right)\right|^{2}} \\
-2 \operatorname{Re}\left(\frac{\hat{\sigma}_{Y_{R} U_{R}, n}^{2}\left(f_{e x}\right)}{\hat{Y}_{R}\left(f_{e x}\right)}\right)
\end{array}\right) .
\end{aligned}
$$

\section{ACKNOWLEDGMENT}

The authors would like to thank the members of the ELEC department of the Vrije Universiteit Brussel for their helpful discussions.
J. A. Pruszynski and S. H. Scott, "Optimal feedback control and the long-latency stretch response," Experimental brain research, vol. 218, pp. 341-359, 2012.

[2] N. Patel, J. Jankovic, and M. Hallett, "Sensory aspects of movement disorders," The Lancet Neurology, vol. 13, pp. 100-112, 2014.

[3] F. Pisano, G. Miscio, C. Del Conte, D. Pianca, E. Candeloro, and R. Colombo, "Quantitative measures of spasticity in post-stroke patients," Clinical Neurophysiology, vol. 111, pp. 1015-1022, 2000.

[4] J. A. Mortimer and D. D. Webster, "Evidence for a quantitative association between EMG stretch responses and parkinsonian rigidity," Brain research, vol. 162, pp. 169-173, 1979.

[5] P. M. Rossini, F. Tecchio, V. Pizzella, D. Lupoi, E. Cassetta, and P. Paqualetti, "Interhemispheric differences of sensory hand areas after monohemispheric stroke: MEG/MRI integrative study," Neuroimage, vol. 14, pp. 474-485, 2001.

[6] J. A. Pruszynski, I. Kurtzer, and S. H. Scott, "The long-latency reflex is composed of at least two functionally independent processes," Journal of neurophysiology, vol. 106, pp. 449-459, 2011.

R. E. Kearney and I. W. Hunter, "System identification of human joint dynamics," Crit Rev Biomed Eng, vol. 18, pp. 55-87, 1990.

A. C. Schouten, E. de Vlugt, J. J. B. van Hilten, and F. C. T. Van der Helm, "Quantifying Proprioceptive Reflexes During Position Control of the Human Arm," Biomedical Engineering, IEEE Transactions on, vol. 55, pp. 311-321, 2008.

M. Mirbagheri, H. Barbeau, M. Ladouceur, and R. Kearney, "Intrinsic and reflex stiffness in normal and spastic, spinal cord injured subjects," Experimental Brain Research, vol. 141, pp. 446459,2001

[10] E. J. Perreault, K. Chen, R. D. Trumbower, and G. Lewis, "Interactions With Compliant Loads Alter Stretch Reflex Gains But Not Intermuscular Coordination," Journal of Neurophysiology, vol. 99, pp. 2101-2113, 2008-05-01 00:00:00 2008.

Sobimatsu, Y. M. Zhang, R. Suga, and M. Kato, "Differential temporal coding of the vibratory sense in the hand and foot in man," Clinical neurophysiology, vol. 111, pp. 398-404, 2000.

C. M. Giabbiconi, C. Dancer, R. Zopf, T. Gruber, and M. M. Müller, "Selective spatial attention to left or right hand flutter sensation modulates the steady-state somatosensory evoked potential," Cognitive brain research, vol. 20, pp. 58-66, 2004.

G. Müller, C. Neuper, and G. Pfurtscheller, "' Resonance-like" frequencies of sensorimotor areas evoked by repetitive tactile stimulation," Biomedizinische Technik. Biomedical engineering, vol. 46, pp. 186-190, 2001.

A. Z. Snyder, "Steady-state vibration evoked potentials: description of technique and characterization of responses," Electroencephalography and Clinical Neurophysiology/Evoked potentials Section, vol. 84, pp. 257-268, 1992.

[15] S. Tobimatsu, Y. M. Zhang, and M. Kato, "Steady-state vibration somatosensory evoked potentials: physiological characteristics and tuning function," Clinical neurophysiology, vol. 110, pp. 1953$1958,1999$.

[16] S. Jamali and B. Ross, "Somatotopic finger mapping using MEG: Toward an optimal stimulation paradigm," Clinical Neurophysiology, vol. 124, pp. 1659-1670, 2013.

A. J. Langdon, T. W. Boonstra, and M. Breakspear, "Multifrequency phase locking in human somatosensory cortex," Progress in biophysics and molecular biology, vol. 105, pp. 58-66, 2011.

S. F. Campfens, A. C. Schouten, M. J. A. M. van Putten, and H. van der Kooij, "Quantifying connectivity via efferent and afferent pathways in motor control using coherence measures and joint position perturbations," Experimental brain research, vol. 228, pp. 141-153, 2013.

[19] W. Mugge, D. A. Abbink, A. C. Schouten, J. P. A. Dewald, and F. C. T. van der Helm, "A rigorous model of reflex function indicates that position and force feedback are flexibly tuned to position and force tasks," Experimental brain research, vol. 200, pp. 325-340, 2010.

\section{REFERENCES}

P. A. Forbes, C. J. Dakin, A. M. Geers, M. P. Vlaar, R. Happee, G. P. Siegmund, et al., "Electrical Vestibular Stimuli to Enhance Vestibulo-Motor Output and Improve Subject Comfort," PLoS ONE, vol. 9, p. e84385, 2014. 
[21] R. Pintelon and J. Schoukens, System Identification: A Frequency Domain Approach: Wiley, 2012.

[22] R. C. Oldfield, "The assessment and analysis of handedness: The Edinburgh inventory," Neuropsychologia, vol. 9, pp. 97-113, 1971.

[23] R. Oostenveld and P. Praamstra, "The five percent electrode system for high-resolution EEG and ERP measurements," Clinical Neurophysiology, vol. 112, pp. 713-719, 4// 2001.

[24] A. C. Schouten, "Proprioceptive reflexes and neurological disorders," Doctoral thesis, Delft University of Technology, Delft, The Netherlands, 2004.

[25] A. C. Schouten, E. de Vlught, and F. C. T. Van der Helm, "Quantification of spinal reflexes in neurological disorders," in Systems, Man and Cybernetics, 2004 IEEE International Conference on, 2004, pp. 2492-2499 vol.3.

[26] J. Schoukens, R. Pintelon, and Y. Rolain, Mastering System Identification in 100 Exercises: Wiley, 2012.

[27] R. Oostenveld, P. Fries, E. Maris, and J.-M. Schoffelen, "FieldTrip: Open Source Software for Advanced Analysis of MEG, EEG, and Invasive Electrophysiological Data," Computational Intelligence and Neuroscience, vol. 2011, p. 9, 2011.

[28] A. C. Schouten, W. Mugge, and F. C. T. van der Helm, "NMClab, a model to assess the contributions of muscle visco-elasticity and afferent feedback to joint dynamics," J Biomech, vol. 41, pp. 165967, 2008.

[29] P. van Drunen, E. Maaswinkel, F. C. T. van der Helm, J. H. van Dieeen, and R. Happee, "Identifying intrinsic and reflexive contributions to low-back stabilization," Journal of biomechanics, vol. 46, pp. 1440-1446, 2013.

[30] M. P. Vlaar, F. C. T. van der Helm, and A. C. Schouten, "Frequency Domain Characterization of the Somatosensory Steady State Response in Electroencephalography," presented at the 17th IFAC Symposium on System Identification SYSID 2015, Beijing, China, 2015.

[31] M. Mirbagheri, H. Barbeau, and R. Kearney, "Intrinsic and reflex contributions to human ankle stiffness: variation with activation level and position," Experimental Brain Research, vol. 135, pp. 423-436, 2000

[32] E. Seiss, C. W. Hesse, S. Drane, R. Oostenveld, A. M. Wing, and P. Praamstra, "Proprioception-Related Evoked Potentials: Origin and Sensitivity to Movement Parameters," NeuroImage, vol. 17, pp. 461-468, 9// 2002.

[33] S. F. Campfens, C. G. M. Meskers, A. C. Schouten, M. J. A. M. van Putten, and H. Van der Kooij, "Stretch evoked potentials in healthy subjects and after stroke: a potential measure for proprioceptive sensorimotor function," Neural Systems and Rehabilitation Engineering, IEEE Transactions on, vol. PP, pp. 1$1,2015$.

[34] J. C. Houk, W. Z. Rymer, and P. E. Crago, Dependence of dynamic response of spindle receptors on muscle length and velocity vol. 46, 1981.

[35] M. P. Mileusnic, I. E. Brown, N. Lan, and G. E. Loeb, Mathematical Models of Proprioceptors. I. Control and Transduction in the Muscle Spindle vol. 96, 2006.
[36] Z. Hasan, "A model of spindle afferent response to muscle stretch," Journal of Neurophysiology, vol. 49, pp. 989-1006, 1983.

[37] M. Dimitriou, "Human Muscle Spindle Sensitivity Reflects the Balance of Activity between Antagonistic Muscles," The Journal of Neuroscience, vol. 34, pp. 13644-13655, 2014.

[38] P. E. Crago, J. C. Houk, and W. Z. Rymer, "Sampling of total muscle force by tendon organs," Journal of Neurophysiology, vol. 47, pp. 1069-1083, 1982.

[39] S. H. Scott, "The computational and neural basis of voluntary motor control and planning," Trends in cognitive sciences, vol. 16, pp. 541-549, 2012.

[40] G. Cruccu, M. J. Aminoff, G. Curio, J. M. Guerit, R. Kakigi, F. Mauguiere, et al., "Recommendations for the clinical use of somatosensory-evoked potentials," Clinical neurophysiology, vol. 119, pp. 1705-1719, 2008.

[41] I. Hashimoto, T. Gatayama, K. Yoshikawa, M. Sasaki, and M. Nomura, "Input-output relation of the somatosensory system for mechanical air-puff stimulation of the index finger in man," Experimental brain research, vol. 88, pp. 645-650, 1992.

[42] O. Franzén and K. Offenloch, "Evoked response correlates of psychophysical magnitude estimates for tactile stimulation in man," Experimental brain research, vol. 8, pp. 1-18, 1969.

[43] Y.-Y. Lin and M. Kajola, "Neuromagnetic somatosensory responses to natural moving tactile stimulation," The Canadian Journal of Neurological Sciences, vol. 30, pp. 31-35, 2003.

[44] D. Johnson, R. Jürgens, and H. H. Kornhuber, "Somatosensoryevoked potentials and vibration," Archiv für Psychiatrie und Nervenkrankheiten, vol. 228, pp. 101-107, 1980.

[45] J. I. Voisin, E. C. Rodrigues, S. Hétu, P. L. Jackson, C. D. Vargas, F. Malouin, et al., "Modulation of the response to a somatosensory stimulation of the hand during the observation of manual actions," Experimental brain research, vol. 208, pp. 11-19, 2011.

[46] C. Nangini, B. Ross, F. Tam, and S. Graham, "Magnetoencephalographic study of vibrotactile evoked transient and steady-state responses in human somatosensory cortex," Neuroimage, vol. 33, pp. 252-262, 2006.

[47] Y. Goto, T. Taniwaki, K.-i. Yamashita, N. Kinukawa, and S. Tobimatsu, "Interhemispheric functional desynchronization in the human vibratory system," Brain Research, vol. 980, pp. 249-254, 8/8/2003.

[48] C. L. Witham, C. N. Riddle, M. R. Baker, and S. N. Baker, "Contributions of descending and ascending pathways to corticomuscular coherence in humans," The Journal of Physiology, vol. 589, pp. 3789-3800, 2011.

[49] L. Narici, K. Portin, R. Salmelin, and R. Hari, "Responsiveness of human cortical activity to rhythmical stimulation: a three-modality, whole-cortex neuromagnetic investigation," NeuroImage, vol. 7, pp. 209-223, 1998. 